Review

\title{
Roles of microRNAs in the resistance to platinum based chemotherapy in the non-small cell lung cancer
}

\author{
Honging Zang ${ }^{1}$, Jianlun Peng², Weiyuan Wang${ }^{1}$, Songqing Fan ${ }^{1 凶}$ \\ 1. Department of Pathology, the Second Xiangya Hospital of Central South University, Changsha, Hunan, 410013, China \\ 2. Department of Pathology, School of Basic Medical Science, Central South University, Changsha, Hunan, 410011, China \\ $\bowtie$ Corresponding author: Songqing Fan, e-mail: songqingfan@csu.edu.cn \\ (C) Ivyspring International Publisher. This is an open access article distributed under the terms of the Creative Commons Attribution (CC BY-NC) license \\ (https://creativecommons.org/licenses/by-nc/4.0/). See http://ivyspring.com/terms for full terms and conditions.
}

Received: 2017.05.31; Accepted: 2017.07.24; Published: 2017.10.17

\begin{abstract}
Platinum-based adjuvant chemotherapy improves survival among patients with lung tumors, in particular non-small cell lung cancer (NSCLC). But the predicament of drug resistance in NSCLC patients is frustrating us. The profiles of microRNAs are different between platinum chemotherapy resistant and sensitive NSCLC cells. Researches regarding microRNAs and their targets, in platinum drug resistant cases, illuminate novel ideals for platinum-based chemotherapy for NSCLC patients. Therefore, in this review we will focus on three aspects: Epithelial-mesenchymal transition (EMT), cell proliferation and apoptosis, and the roles of microRNAs in cisplatin (CDDP) and carboplatin (CBP) resistance.
\end{abstract}

Key words: microRNAs; platinum drugs; cisplatin; carboplatin; drug resistance; non-small cell lung cancer

\section{Introduction}

Non-small cell lung cancer (NSCLC), as its name suggests, refers to all types of lung cancers except small cell lung cancer (SCLC). NSCLC encompasses the majority of lung cancers, and is the leading cause of cancer related deaths worldwide ${ }^{1}$. For NSCLC patients in clinical stage II-III with completely surgical removal of tumor, adjuvant cisplatin (CDDP, cis-diammine-dichloro-platinum II) based chemotherapy is recommended, when driver mutation hasn't been detected ${ }^{1}$. CDDP, approved by the FDA in 1978, is a traditional cytotoxic chemotherapy drug for types of solid tumors, especially NSCLC2. It forms cisplatin-DNA adducts to destroy functions of DNA leading to the cytotoxicity ${ }^{3}$. Unfortunately, CDDP resistance constitutes the current therapeutic plateau which needs to be overcome. This drug resistance is classified into two types: primary resistance which refers to chemoresistance before chemotherapy, and acquired resistance which occurs after chemotherapy². Dysregulations of drug transport and/or metabolism, targeting oncogenes and inhibited drug-induced apoptosis are common mechanisms of drug resistance ${ }^{4}$. Carboplatin (CBP) is the second generation platinum medicine which is cis-diammine (1, 1-cyclobutanedicarboxylatoplatinum (II)), and produces less ototoxicity and nephrotoxicity ${ }^{1}$. Unfortunately, there is cross resistance between CDDP and CBP. Though CBP is also widely used in NSCLC, the primary and secondary resistance to it still confounds patients and doctors. Oxaliplatin is [(1R, 2R)-cyclohexane-1, 2-diamine] (ethanedioato-O, $O^{\prime}$ ) platinum (II), and it's often used to treating colorectal cancer ${ }^{2}$. Nedaplatin is cis-diamineglycolate-O, $\mathrm{O}^{\prime}$-platinum (II) ${ }^{2}$. However, there is little researches regarding these two drugs resistance to NSCLC, therefore we will not discuss these platinum medicines in this article.

MicroRNAs are endogenous small non-coding RNAs, usually 20-22nt. This class of RNA regulates numerous biological processes via negatively regulating RNA expression ${ }^{5}$. Recently, scientists have demonstrated that microRNAs modulate drug resistance in many such as diffuse large $B$ cell lymphoma and gastric cancer ${ }^{6-7}$. Prior most researches have also demonstrated the roles of microRNAs in the NSCLC drug resistance. Our team has summarized microRNAs that modulate NSCLC-targeted 
treatments, and found that different microRNAs could exert opposite influence in certain target medicine clinical pharmacotherapy ${ }^{8}$. In this review, we discuss the roles of microRNAs in platinum agent resistance and also the possible mechanisms, which provide us a new point of penetration to improve treatments of NSCLC, especially for patients without indications for targeted medicine like EGFR-TKIs (epidermal growth factor receptor-tyrosine kinase inhibitors).

\section{CDDP resistance}

According to the latest clinical guidelines for the treatment of NSCLC, CDDP-based chemotherapy is recommended for the majority of early stage and all advanced NSCLC patients without ALK/EGFR mutation ${ }^{1}$. Nevertheless the efficacy is impeded by primary resistance, or acquired resistance which usually occurs early. Recent studies have demonstrated that silencing or activating certain miRNAs could reverse drug resistance through different mechanisms.

\section{CDDP resistance and epithelial-mesenchymal transition (EMT)}

EMT indicates the phenotypical alterations that occur when epithelial cells lose the epithelial characteristics such as the polarization and cell junctions, and acquire mesenchymal phenotypes ${ }^{9}$. In recent studies, EMT is found to be closely related with chemoresistance rather than metastasis in solid tumors like lung cancer and pancreatic cancer-10. Moreover, a study in oral squamous cell carcinoma (OSCC) has revealed that some miRNAs modulate CDDP resistance partially through regulating EMT ${ }^{11}$. In NSCLC, microspherule protein 1 (MCRS1), specifically negatively regulated by miR-129* (miR-129-1-3p), induces EMT and CDDP resistance, through increasing the expression of miR-15512. Paolo Ceppi et al. found that miR-200c inhibited CDDP and cetuximab resistance, with a significant increase of E-cadherin and a decreased of $\mathrm{N}$-cadherin expression which plays an important role in regulating EMT $^{13}$.There is evidence that miR-17 family $(-17,20 a$, $20 \mathrm{~b})$, through negatively regulating TGF-beta receptor type-2(TGFbR2), induces EMT and CDDP resistance ${ }^{14}$.

MiR-15b, directly targeting phosphatidylethanolamine-binding protein 4(PEBP4), can both promote CDDP resistance and EMT ${ }^{15}$. Raf Kinase Inhibitory Protein (RKIP), targeted by mir-27a can inhibit EMT and CDDP resistance ${ }^{16}$. MiR-181a, which is remarkably upregulated in the CDDP-resistant A549 cell line than in the parental cell line, promotes EMT by targeting phosphatase and tensin homolog deleted on chromosome 10 (PTEN), but the role of
miR-181a in CDDP resistance will not be discussed in this study ${ }^{17}$. Interestingly, in another study, PTEN, targeted by mir-92b could suppress CDDP-induced apoptosis $^{18}$. Taken together, EMT may directly contribute to CDDP resistance via PTEN. (MicroRNAs and their targets mentioned before are summarized in Table 1).

In conclusion, EMT is an important factor in the biological behaviors of miRNAs regulating CDDP resistance in NSCLC, and provides a novel future for treatments of CDDP resistant patients. Nevertheless, this hypothesis needs more supporting evidence.

Table 1. miRNAs involved in CDDP resistance and EMT in NSCLC

\begin{tabular}{llll}
\hline MiRNAs & Targets & Targeted by & References \\
\hline MiR-129*(miR-129-1-3p) & MCRS1 & $/$ & {$[12]$} \\
MiR-155 & $/$ & MCRS & {$[12]$} \\
MiR-200c & E-cadherin, & $/$ & {$[13]$} \\
& N-cadherin\# & & \\
MiR-17 family $(-17,20 a$, & TGFbR2 & $/$ & {$[14]$} \\
20b) & & & \\
MiR-15b & PEBP4 & $/$ & {$[15]$} \\
MiR-27 & RIKP & $/$ & {$[16]$} \\
MiR-181a & PTEN & $/$ & {$[17]$} \\
\hline \#: MiR-200c restored E-cadherin expression ${ }^{13 ; *}:$ MiR-129* indicates miR-129-1-3; /: \\
no mention in the references.
\end{tabular}

\section{Cisplatin resistance and cell proliferation}

Uncontrolled cell proliferation is one of the important classic characteristics of tumors. We want to illuminate whether some miRNAs achieve the resistance or opposite effects through acting on cell cycle. Jian Zhao et al. have found that miR-17 family $(-17,20 a, 20 b)$ arrests cell cycle at G1/S phase through inhibition of CDKN1A, and miR-17 family can reverse CDDP resistance ${ }^{19}$. Additionally, expressions of miR-17 and miR-92 families are related to responses of patients to CDDP treatments without deeper investigations ${ }^{19}$. MiR-217, inhibiting cell proliferation, reverses CDDP resistance through targeting Kirsten rat sarcoma viral oncogene homolog (KRAS) gene ${ }^{20}$. MiR-26a, which is down-regulated in CDDP-resistant A549 cell lines, promotes cell sensitivity to CDDP through inhibiting cell growth by targeting the High Mobility Group A 2(HMGA2)-mediated E2F1-Akt pathway ${ }^{21}$. P53 directly modulates miR-34a, and in turn miR-34a contributes to thep53 network for tumor suppression especially inhibiting tumor cell proliferation $22-24$. PEBP4, targeted by miR-34a, could promote CDDP resistance, meanwhile PEBP4 can inactivate $\mathrm{p} 53^{25}$. In short, it will re-sensitize CDDP-resistant in NSCLC to CDDP when activating the p53/miR-34a loop. Further investigations into the pharmacological mechanisms are still ongoing.

MiR-1 reverses CDDP resistance via inhibiting 
SDF-1 and CAFS on cell proliferation ${ }^{26}$. And miR-138 targets cyclin D3 (CCND3) to regulate cell proliferation ${ }^{27}$. (MicroRNAs and their targets mentioned previously are summarized in Table 2).

In summary, microRNAs could influence tumor cell proliferation to promote or reverse CDDP resistance. These microRNAs and their targets provide novel insights for treatments and prognostic predictions.

Table 2. miRNAs involved in CDDP resistance and cell proliferation in NSCLC

\begin{tabular}{llll}
\hline MiRNAs & Targets & Targeted by & References \\
\hline $\begin{array}{l}\text { MiR-17 family } \\
(-17,20 a, 20 b)\end{array}$ & CDKN1A & $/$ & {$[19]$} \\
MiR-217 & KRAS & $/$ & {$[20]$} \\
MiR-26a & HMGA2-mediated & $/$ & {$[21]$} \\
& E2F1-Akt pathway & & \\
MiR-34a & PEBP4 & P53 & {$[22][25]$} \\
MiR-1 & SDF-1,CAFS & $/$ & {$[26]$} \\
MiR-138 & CCND3 & $/$ & {$[27]$} \\
\hline
\end{tabular}

/: no mention in the references.

\section{CDDP resistance and cell apoptosis}

Apoptosis is the progression of cell-programmed death and naturally suppresses tumor progression ${ }^{28}$. Though defects in apoptosis of tumor cells contribute to drug resistance, resulting in the failure of anti-cancer therapy, multiple microRNAs have been demonstrated to participate in cell apoptosis ${ }^{29-30}$. Better understanding of correlations between microRNAs and apoptosis in NSCLC may provide basic principles to improve current therapies.

The cellular uptake of CDDP as a cytotoxic chemotherapy drug triggers some cellular pathways and causes cytotoxic effects ${ }^{3}$. The ATP-binding cassette $(A B C)$ transporter superfamily is regarded as the largest transporter family, encompassing seven subfamilies (ABCA ABCG). Previous studies have found $A B C B$ and $A B C C$ subfamilies are involved in multiple drug resistances ${ }^{4}$. Also, several miRNAs can regulate certain $A B C$ transporters which influence CDDP influx or efflux to impair CDDP-induced apoptosis in NSCLC. ABCB9 works as a drug transporter, while miR-31 induces ABCB9 mRNA degradation to decrease CDDP-induced apoptosis through decreased CDDP uptake ${ }^{31}$. Let-7c can reverse $\mathrm{CDDP}$ resistance by targeting $\mathrm{ABCC} 2^{32}$. Additionally, ABCA1, targeted by miR-106a, can transport CDDP into cells ${ }^{33}$.

Besides drug transporters, miRNAs also target other signals to induce or inhibit apoptosis of NSCLC cells. Apoptotic pathways are commonly classified into intrinsic and extrinsic pathways. Bcl-2 family members participate in the intrinsic apoptotic pathway, and different members may have opposite functions $^{30}$. MiR-503 and miR-497 target the anti-apoptotic $\mathrm{Bcl}-2$ gene to reverse CDDP resistance $^{34-35}$, and conversely miR-21 decreases expression of anti-apoptotic Bax and increases pro-apoptotic Bcl-2 resulting in re-acquiring CDDP sensitivity $^{36}$.

Decreased miR-184 could confer CDDP resistance via enhancing $\mathrm{Bcl}-2^{37}$. Bim is a member of the Bcl-2 family that as the opposite effect of Bcl-2. The intrinsic apoptotic pathway relies on the apoptosome, composed of apoptotic protease activating factor 1 (Apaf-1), cytochrome $\mathrm{c}$ and procaspase-930. MiR-155 targeting Apaf-1 and miR-137 targeting caspase-3(CASP3) promote CDDP resistance in lung cancer $38-39$. Mir-940 and mir-92b could suppress CDDP-induced apoptosis by respectively targeting mitogen-activated protein kinase phosphatase-1 (MKP1) and PTEN18, 40. MiR-138, in addition to the functions in cell cycle discussed before, also could promote CDDP-induced apoptosis by targeting (excision repair cross-complementation group 1) ERCC1 ${ }^{41}$.

EGCG enhances CDDP efficacy in apoptosis in NSCLC by inhibiting miR-98-5 $\mathrm{p}^{42-43}$. MiR-218 can reverse CDDP resistance by decreasing runt-related transcription factor 2(RUNX2) ${ }^{44}$. MiR-1244 may enhance CDDP resistance probably by targeting TP5345. MiR-196a may confer CDDP resistance via inhibiting apoptosis probably by targeting human multiple drug resistance 1 (MDR1), multidrug resistance protein 1 (MRP1), excision repair cross-complementation 1 (ERCC1), survivin, and B cell lymphoma 2 (Bcl-2) ${ }^{46,47}$. (MicroRNAs and their targets mentioned previously are summarized in Table 3).

Table 3. miRNAs involved in CDDP resistance and apoptosis in NSCLC

\begin{tabular}{llll}
\hline MiRNAs & Targets & $\begin{array}{l}\text { Targeted } \\
\text { by }\end{array}$ & References \\
\hline MiR-31 & ABCB9 & $/$ & {$[31]$} \\
Let-7c & ABCC2 & $/$ & {$[32]$} \\
MiR-106a & ABCA1 & $/$ & {$[33]$} \\
MiR-503 & Bcl-2 & $/$ & {$[34]$} \\
MiR-497 & Bcl-2 & $/$ & {$[35]$} \\
MiR-21 & Bax & $/$ & {$[36]$} \\
MiR-184 & Bcl-2 & $/$ & {$[37]$} \\
MiR-155 & Apaf-1 & $/$ & {$[38]$} \\
MiR-137 & CASP3 & $/$ & {$[39]$} \\
Mir-940 & MKP1 & $/$ & {$[40]$} \\
MiR-92b & PTEN & $/$ & {$[18]$} \\
MiR-138 & $/$ & EERCC1 & {$[41]$} \\
MiR-98-5p & EGCG & $/$ & {$[42][43]$} \\
MiR-218 & RUNX2 & $/$ & {$[44]$} \\
MiR-1244 & TP53 & $/$ & {$[45]$} \\
MiR-196 & MDR1,MRP1,ERCC1,survivin, Bcl-2 / & {$[46][47]$} \\
\hline
\end{tabular}

/: no mention in the references. 


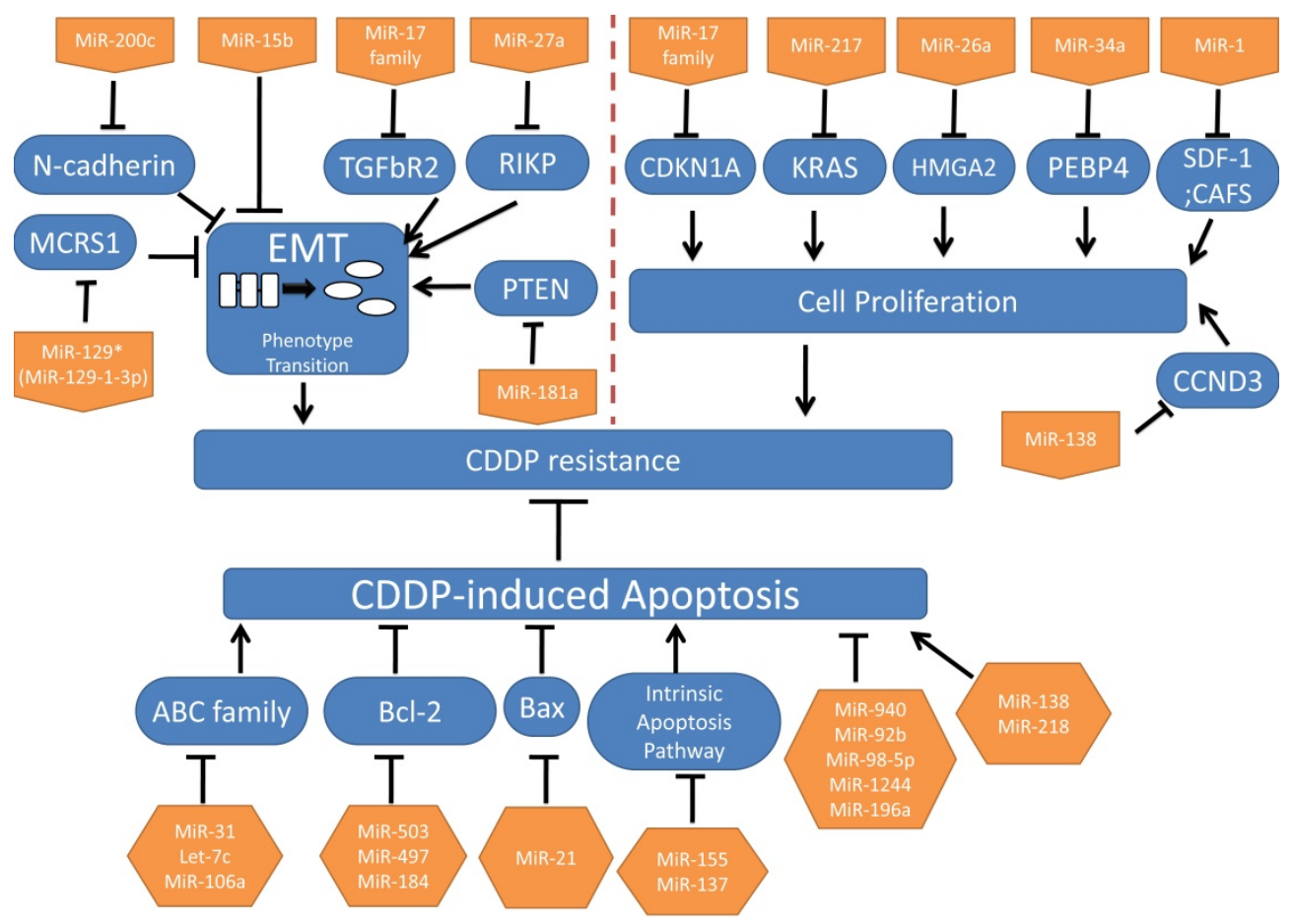

Figure 1. MicroRNAs involved in CDDP resistance in NSCLC.

In conclusion, we primarily summarized microRNAs that appear to be important in CDDP resistance from different biological processions: EMT, cell proliferation and apoptosis. (These microRNAs are summarized in Figure 1.)

\section{Carboplatin(CBP)}

There are similar anti-tumor mechanisms between CDDP and CBP, which has fewer side-effects to the kidneys and nervoussystem ${ }^{48}$. There're few researches regarding microRNAs and CBP in lung cancers. Only miR-218 and miR-205 were found to have opposite functions in $\mathrm{CBP}$ resistance in NSCLC ${ }^{49}$. MiR-218 reverses CBP resistance by stimulating apoptosis and cell cycle arrest, while miR-205 promotes cell proliferation to achieve the inverse effects ${ }^{49}$.

In ovarian cancer, miR-21, miR-214, miR-193b, miR-200c and miR-141 were related with platinum resistance ${ }^{50-52}$. MiR-21 and miR-214 were over-expressed in the ascites cells which were resistant to CBP therapy compared to omental metastases ${ }^{50}$, while miR-21 was found to be inhibited by carboplatin and increase the cell invasion. Further studies on miR-193b, miR-200c and miR-141 show these miRNAs conferred resistance to CBP in ovarian cell lines ${ }^{51-52}$.

In breast cancer, miR-664b-5p increases CBP with gemcitabine sensitivity via targeting CCNE2 ${ }^{53}$. MiR-222/ 223 in mesenchymal stem cell-derived exosome increases CBP resistance which needs further research ${ }^{54}$. MiR-621 sensitizes breast cancer cells to PTX/CBP by inhibiting FBXO11 and activatingp5355. Patients with overexpressed miR-659-3p predicted complete or partial response to $\mathrm{CBP} /$ paclitaxel-based treatments ${ }^{56}$.

Although little attention was paid to the roles of miRNAs in CBP resistant NSCLC (from Figure 2) they may be important in predicating CBP sensitivity and acting as a novel targeting mechanism for reversing resistance.

\section{Conclusion}

Lung cancer is usually recognized late and is prevalent in cancer related death. NSCLC contributes for the most of lung cancer, and the majority of NSCLC patients are recommended CDDP-based chemotherapy, but CDDP resistance occurs sooner than anticipated. Therefore, we aim to find a new method to reverse CDDP and/or CBP resistance to assist NSCLC patients.

MicroRNAs operate numerous biological signal pathways through negatively modulating expressions of targeted genes. Researchers have demonstrated the importance of microRNAs in CDDP resistance in NSCLC patients. In this review, we classified functions of microRNAs in CDDP resistance into three classes: Class A microRNAs regulate both CDDP resistance and EMT in NSCLC, like miR-129*(miR-129-1-3p), miR-200c, miR-17 family 


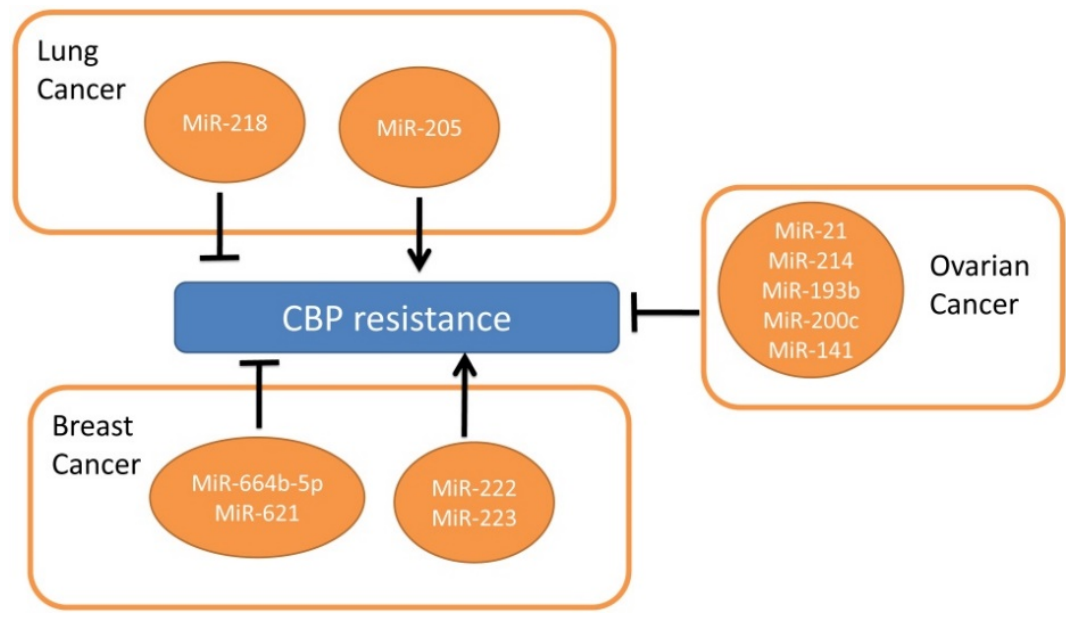

Figure 2. MicroRNAs involved in CBP resistance.

human participants or animals performed by any of the authors.

\section{Competing Interests}

The authors have declared that no competing interest exists.

\section{References}

1. Garcia-Campelo R, Bernabe R, Cobo M, Corral J, Coves J, Domine M, et al. SEOM clinical guidelines for the treatment of non-small cell lung cancer (NSCLC) 2015. Clinical \& translational oncology: official publication of the Federation of Spanish Oncology Societies and of the National Cancer Institute of Mexico. 2015; 17: 1020-9.

2. Kelland L. The resurgence of platinum-based cancer chemotherapy. Nature reviews Cancer. 2007; 7: 573-84.

3. Wang D, Lippard SJ. Cellular processing of platinum anticancer drugs. Nature reviews Drug discovery. 2005; 4: $307-20$

4. Hamilton G, Rath B. A short update on cancer chemoresistance. Wiener medizinische Wochenschrift (1946). 2014; 164: 456-60.

5. Bartel DP. MicroRNAs: genomics, biogenesis,

$(-17,20 a, 20 b)$, miR-15b, miR-27 and miR181a ${ }^{12-17}$; Class B includes microRNAs which regulate cell proliferation to contribute to CDDP resistance or sensitivity, such as the miR-17 family $(-17,20 a, 20 b)$, miR-217, miR-26a, miR-34a,miR-1, miR-26a, and miR-138(19-23,25,26-27); Class C contains CDDP resistance related microRNAs which coordinate apoptosis of NSCLC cells, such as miR-31, Let-7c, miR-106a, miR-503, miR-497, miR-21, miR-184, miR-155, miR-137, mir-940, miR-92b and miR-138, miR-98-5p,miR-218, miR-1244, and miR-19618,31-38,40-45. Distinctions between the three classes are not absolute: for example, miR-34a reverses CDDP resistance and inhibits EMT, but also inhibits cell proliferation $22-25$.

Regarding CBP resistance in NSCLC, miR-218 and miR-205 are the only two microRNAs we found in studies which have opposite roles in CBP resistance ${ }^{49}$, while microRNAs functions in CBP resistance in other tumors are widely studied. There are bright prospects for the study and utilization of microRNAs in reversing CBP resistance.

Overall, further understanding of microRNAs and their targets in CDDP and CBP resistance may contribute to NSCLC treatments. Safely and efficiently increasing the expression of advantageous microRNAs and decreasing expression of disadvantageous microRNAs, although challenging, may provide welcome developments through further study of their targets and mechanisms.

\section{Acknowledgments}

This work was supported by the National Natural Science Foundations of China (No: 81272566; 81472773 and 81773218).

\section{Ethical approval}

This article does not contain any studies with mechanism, and function. Cell. 2004; 116: 281-97.

6. Yu X, Li Z. New insights into MicroRNAs involves in drug resistance in diffuse large B cell lymphoma. American journal of translational research. 2015; 7: 2536-42.

7. Riquelme I, Letelier P, Riffo-Campos AL, Brebi P, Roa JC. Emerging Role of miRNAs in the Drug Resistance of Gastric Cancer. International journal of molecular sciences. 2016; 17.

8. Zang $\mathrm{H}$, Wang $\mathrm{W}$, Fan $\mathrm{S}$. The role of microRNAs in resistance to targeted treatments of non-small cell lung cancer. Cancer chemotherapy and pharmacology. 2017; 79(2):227-231.

9. Fischer KR, Durrans A, Lee S, Sheng J, Li F, Wong ST, et al. Epithelial-to-mesenchymal transition is not required for lung metastasis but contributes to chemoresistance. Nature. 2015; 527: 472-6.

10. Zheng X, Carstens JL, Kim J, Scheible M, Kaye J, Sugimoto H, et al. Epithelial-to-mesenchymal transition is dispensable for metastasis but induces chemoresistance in pancreatic cancer. Nature. 2015; 527: 525-30.

11. Ghosh RD, Ghuwalewala S, Das P, Mandloi S, Alam SK, Chakraborty J, et al. MicroRNA profiling of cisplatin-resistant oral squamous cell carcinoma cell lines enriched with cancer-stem-cell-like and epithelial-mesenchymal transition-type features. Scientific reports. 2016; 6: 23932.

12. Liu MX, Zhou KC, Cao Y. MCRS1 overexpression, which is specifically inhibited by miR-129*, promotes the epithelial-mesenchymal transition and metastasis in non-small cell lung cancer. Molecular cancer. 2014; 13: 245.

13. Ceppi P, Mudduluru G, Kumarswamy R, Rapa I, Scagliotti GV, Papotti M, et al. Loss of miR-200c expression induces an aggressive, invasive, and chemoresistant phenotype in non-small cell lung cancer. Molecular cancer research: MCR. 2010; 8: 1207-16.

14. Jiang Z, Yin J, Fu W, Mo Y, Pan Y, Dai L, et al. MiRNA 17 family regulates cisplatin-resistant and metastasis by targeting TGFbetaR2 in NSCLC. PloS one. 2014; 9: e94639.

15. Zhao Z, Zhang $L, Y$ ao $Q$, Tao Z. miR-15b regulates cisplatin resistance and metastasis by targeting PEBP4 in human lung adenocarcinoma cells. Cancer gene therapy. 2015; 22: 108-14.

16. Li J, Wang Y, Song Y, Fu Z, Yu W. miR-27a regulates cisplatin resistance and metastasis by targeting RKIP in human lung adenocarcinoma cells. Molecular cancer. 2014; 13: 193

17. Li H, Zhang P, Sun X, Sun Y, Shi C, Liu H, et al. MicroRNA-181a regulates epithelial-mesenchymal transition by targeting PTEN in drug-resistant lung adenocarcinoma cells. International journal of oncology. 2015; 47: 1379-92.

18. Li Y, Li L, Guan Y, Liu X, Meng Q, Guo Q. MiR-92b regulates the cell growth, cisplatin chemosensitivity of A549 non small cell lung cancer cell line and target PTEN. Biochemical and biophysical research communications. 2013; 440: 604-10.

19. Zhao J, Fu W, Liao H, Dai L, Jiang Z, Pan Y, et al. The regulatory and predictive functions of miR-17 and miR-92 families on cisplatin resistance of non-small cell lung cancer. BMC cancer. 2015; 15: 731 .

20. Guo J, Feng Z, Huang Z, Wang H, Lu W. MicroRNA-217 functions as a tumour suppressor gene and correlates with cell resistance to cisplatin in lung cancer. Molecules and cells. 2014; 37: 664-71.

21. Yang Y, Zhang P, Zhao Y, Yang J, Jiang G, Fan J. Decreased MicroRNA-26a expression causes cisplatin resistance in human non-small cell lung cancer. Cancer biology \& therapy. 2016; 17: 515-25.

22. Kim NH, Kim HS, Li XY, Lee I, Choi HS, Kang SE, et al. A p53/miRNA-34 axis regulates Snail1-dependent cancer cell epithelial-mesenchymal transition. The Journal of cell biology. 2011; 195: 417-33.

23. He L, He X, Lim LP, de Stanchina E, Xuan Z, Liang Y, et al. A microRNA component of the p53 tumour suppressor network. Nature. 2007; 447: 1130-4. 
24. Bommer GT, Gerin I, Feng Y, Kaczorowski AJ, Kuick R, Love RE, et al. p53-mediated activation of miRNA34 candidate tumor-suppressor genes. Current biology: CB. 2007; 17: 1298-307.

25. Yu G, Zhong N, Chen G, Huang B, Wu S. Downregulation of PEBP4, a target of miR-34a, sensitizes drug-resistant lung cancer cells. Tumour biology: the journal of the International Society for Oncodevelopmental Biology and Medicine. 2014; 35: 10341-9.

26. Li J, Guan J, Long X, Wang Y, Xiang X. mir-1-mediated paracrine effect of cancer-associated fibroblasts on lung cancer cell proliferation and chemoresistance. Oncology reports. 2016; 35: 3523-31.

27. Han LP, Fu T, Lin Y, Miao JL, Jiang QF. MicroRNA-138 negatively regulates non-small cell lung cancer cells through the interaction with cyclin D3. Tumour biology: the journal of the International Society for Oncodevelopmental Biology and Medicine. 2016; 37: 291-8.

28. Paul I, Jones JM. Apoptosis block as a barrier to effective therapy in non small cell lung cancer. World journal of clinical oncology. 2014; 5: 588-94.

29. Johnstone RW, Ruefli AA, Lowe SW. Apoptosis: a link between cancer genetics and chemotherapy. Cell. 2002; 108: 153-64.

30. Su Z, Yang Z, Xu Y, Chen Y, Yu Q. MicroRNAs in apoptosis, autophagy and necroptosis. Oncotarget. 2015; 6: 8474-90.

31. Dong Z, Zhong Z, Yang L, Wang S, Gong Z. MicroRNA-31 inhibits cisplatin-induced apoptosis in non-small cell lung cancer cells by regulating the drug transporter ABCB9. Cancer letters. 2014; 343: 249-57.

32. Zhan M, Qu Q, Wang G, Zhou H. Let-7c sensitizes acquired cisplatin-resistant A549 cells by targeting ABCC2 and Bcl-XL. Die Pharmazie. 2013; 68: 955-61.

33. Ma Y, Li X, Cheng S, Wei W, Li Y. MicroRNA-106a confers cisplatin resistance in non-small cell lung cancer A549 cells by targeting adenosine triphosphatase-binding cassette A1. Molecular medicine reports. 2015; 11: 625-32.

34. Qiu T, Zhou L, Wang T, Xu J, Wang J, Chen $\mathrm{W}$, et al. miR-503 regulates the resistance of non-small cell lung cancer cells to cisplatin by targeting Bcl-2. International journal of molecular medicine. 2013; 32: 593-8.

35. Zhu W, Zhu D, Lu S, Wang T, Wang J, Jiang B, et al. miR-497 modulates multidrug resistance of human cancer cell lines by targeting BCL2. Medical oncology. 2012; 29: 384-91.

36. Xu L, Huang Y, Chen D, He J, Zhu W, Zhang Y, et al. Downregulation of miR-21 increases cisplatin sensitivity of non-small-cell lung cancer. Cancer genetics. 2014; 207: 214-20.

37. Tung MC, Lin PL, Cheng YW, Wu DW, Yeh SD, Chen CY, et al. Reduction of microRNA-184 by E6 oncoprotein confers cisplatin resistance in lung cancer via increasing Bcl-2. Oncotarget. 2016; 7: 32362-74.

38. Zang YS, Zhong YF, Fang Z, Li B, An J. MiR-155 inhibits the sensitivity of lung cancer cells to cisplatin via negative regulation of Apaf-1 expression. Cancer gene therapy. 2012; 19: 773-8.

39. Su TJ, Ku WH, Chen HY, Hsu YC, Hong OS, Chang GC, et al. Oncogenic miR-137 contributes to cisplatin resistance via repressing CASP3 in lung adenocarcinoma. American journal of cancer research. 2016; 6: 1317-30.

40. Wang Q, Shi S, He W, Padilla MT, Zhang L, Wang X, et al. Retaining MKP1 expression and attenuating JNK-mediated apoptosis by RIP1 for cisplatin resistance through miR-940 inhibition. Oncotarget. 2014; 5: 1304-14.

41. Wang Q, Zhong M, Liu W, Li J, Huang J, Zheng L. Alterations of microRNAs in cisplatin-resistant human non-small cell lung cancer cells (A549/DDP). Experimental lung research. 2011; 37: 427-34

42. Zhou DH, Wang $X$, Feng Q. EGCG enhances the efficacy of cisplatin by downregulating hsa-miR-98-5p in NSCLC A549 cells. Nutrition and cancer. 2014; 66: 636-44.

43. Jiang $P, W u X$, Wang $X$, Huang $W$, Feng Q. NEAT1 upregulates EGCG-induced CTR1 to enhance cisplatin sensitivity in lung cancer cells. Oncotarget. 2016; 7: 43337-51

44. Xie J, Yu F, Li D, Zhu X, Zhang X, Lv Z. MicroRNA-218 regulates cisplatin (DPP) chemosensitivity in non-small cell lung cancer by targeting RUNX2. Tumour biology: the journal of the International Society for Oncodevelopmental Biology and Medicine. 2016; 37: 1197-204.

45. Li W, Wang W, Ding M, Zheng X, Ma S, Wang X. MiR-1244 sensitizes the resistance of non-small cell lung cancer A549 cell to cisplatin. Cancer cell international. 2016; 16: 30.

46. Li JH, Luo N, Zhong MZ, Xiao ZQ, Wang JX, Yao XY, et al. Inhibition of microRNA-196a might reverse cisplatin resistance of A549/DDP non-small-cell lung cancer cell line. Tumour biology: the journal of the International Society for Oncodevelopmental Biology and Medicine. 2016; 37: 2387-94.

47. Li Q, Yang Z, Chen M, Liu Y. Downregulation of microRNA-196a enhances the sensitivity of non-small cell lung cancer cells to cisplatin treatment. International journal of molecular medicine. 2016; 37: 1067-74.

48. Thomas JP, Lautermann J, Liedert B, Seiler F, Thomale J. High accumulation of platinum-DNA adducts in strial marginal cells of the cochlea is an early event in cisplatin but not carboplatin ototoxicity. Molecular pharmacology. 2006; 70: 23-9.

49. Zarogoulidis $\mathrm{P}$, Petanidis $\mathrm{S}$, Kioseoglou $\mathrm{E}$, Domvri $\mathrm{K}$ Anestakis $\mathrm{D}$ Zarogoulidis K. MiR-205 and miR-218 expression is associated with carboplatin chemoresistance and regulation of apoptosis via Mcl-1 and Survivin in lung cancer cells. Cellular signalling. 2015; 27: 1576-88.

50. Frederick PJ, Green HN, Huang JS, Egger ME, Frieboes HB, Grizzle WE, et al. Chemoresistance in ovarian cancer linked to expression of microRNAs.
Biotechnic\&histochemistry: official publication of the Biological Stain Commission. 2013; 88: 403-9.

51. Ziliak D, Gamazon ER, Lacroix B, Kyung Im H, Wen Y, Huang RS. Genetic variation that predicts platinum sensitivity reveals the role of miR-193b* in chemotherapeutic susceptibility. Molecular cancer therapeutics. 2012; 11: 2054-61.

52. Brozovic A, Duran GE, Wang YC, Francisco EB, Sikic BI. The miR-200 family differentially regulates sensitivity to paclitaxel and carboplatin in human ovarian carcinoma OVCAR-3 and MES-OV cells. Molecular oncology. 2015; 9: 1678-93.

53. Song $\mathrm{W}$, Tang $\mathrm{L}, \mathrm{Xu} \mathrm{Y}, \mathrm{Xu}$ J, Zhang $\mathrm{W}$, Xie H, et al. PARP inhibitor increases chemosensitivity by upregulating miR-664b-5p in BRCA1-mutated triple-negative breast cancer. Scientific reports. 2017; 7: 42319.

54. Bliss SA, Sinha G, Sandiford OA, Williams LM, Engelberth DJ, Guiro K, et al. Mesenchymal Stem Cell-Derived Exosomes Stimulate Cycling Quiescence and Early Breast Cancer Dormancy in Bone Marrow. Cancer research. 2016; 76: 5832-44

55. Xue J, Chi Y, Chen Y, Huang S, Ye X, Niu J, et al. MiRNA-621 sensitizes breast cancer to chemotherapy by suppressing FBXO11 and enhancing p53 activity. Oncogene. 2016; 35: 448-58.

56. Villaruz LC, Huang G, Romkes M, Kirkwood JM, Buch SC, Nukui T, et al. MicroRNA expression profiling predicts clinical outcome of carboplatin/paclitaxel-based therapy in metastatic melanoma treated on the ECOG-ACRIN trial E2603. Clinical epigenetics. 2015; 7: 58. 